\title{
Involvement of the corpus callosum splenium in a case with SSPE: magnetic resonance spectroscopy findings
}

Alpay Alkan ${ }^{1}$, Rasul Sharifov', Serhat Guler ${ }^{2}$, Ayse Aralasmak', Abdulkadir Kocer ${ }^{3}$, Adnan Yuksel ${ }^{2}$

1Department of Radiology, Bezmialem Vakif University School of Medicine, İstanbul,
Turkey
2Department of Pediatric Neurology, Bezmialem Vakif University School of Medicine,
İstanbul, Turkey
3Department of Neurology, Bezmialem Vakif University School of Medicine, İstanbul,
Turkey

Submitted: 16 October 2012

Accepted: 9 November 2012

Arch Med Sci 2013; 9, 2: 386-387

DOI: 10.5114/aoms.2013.34420

Copyright @ 2013 Termedia \& Banach

Subacute sclerosing panencephalitis (SSPE) is a fatal, incurable, inflammatory, and neurodegenerative disease. The parieto-occipital lobes are the most frequently and severely affected. The basal ganglia, cerebellum, spinal cord and corpus callosum are less frequently involved [1-4]. Because magnetic resonance spectroscopy (MRS) provides information regarding in vivo brain metabolism and neuronal function, it is a promising method that may illustrate the pathophysiological features of SSPE [5-8]. To our knowledge, this is the first report of MRS findings of corpus callosum in a case with SSPE.

A 15-year-old, previously healthy boy was hospitalized because of 4-week history of muscle weakness, impaired vision, and gait imbalance. He had not been vaccinated. A neurological examination revealed symmetric muscular weakness, ataxia, sequential slow movement, decreased motor activity and deep tendon reflexes, and negative Babinski reflex. He had exhibited myoclonic jerks, intellectual deterioration and behavioral changes. The characteristic burst suppression pattern was seen on EEG. Cerebrospinal fluid investigation revealed oligoclonal IgG bands and elevated titers of the measles antibody. He was diagnosed as clinical stage II according to the Jabbour classification.

Magnetic resonance imaging (MRI) demonstrated diffuse hyperintensity in the cortical gray and subcortical, deep and periventricular white matter of the parieto-occipital region with mass effect observed as sulcal effacement and hyperintensity in the splenium of the corpus callosum on T2 and FLAIR weighted images. MRS was performed (TE: $135 \mathrm{~ms}$ ). Voxels were placed in the right parietooccipital region and splenium of the corpus callosum. MR spectrum obtained from right parietooccipital subcortical and deep white matter and splenium of the corpus callosum showed a significant decrease in $\mathrm{N}$-acetylaspartate (NAA)/creatine ( $\mathrm{Cr}$ ) and increase in the ratio of choline (Cho)/Cr. Additionally, there were also inverted prominent lactate peaks (Figures 1 and 2).

Subacute sclerosing panencephalitis is persistent and chronic encephalitis secondary to measles virus infection that causes widespread demyelination of the central nervous system. Since clinical profiles of the disease lead to various presentations, early diagnosis and true clinical staging are not always easy. Usually, MRI findings and the clinical stage of SSPE are

\author{
Corresponding author: \\ Alpay Alkan MD \\ Department of Radiology \\ School of Medicine \\ Bezmialem Vakif University \\ Vatan Street, Aksaray \\ İstanbul, Turkey \\ Phone: 902124531700 \\ Fax: 902126318551 \\ E-mail: \\ alpay_alkan@hotmail.com
}




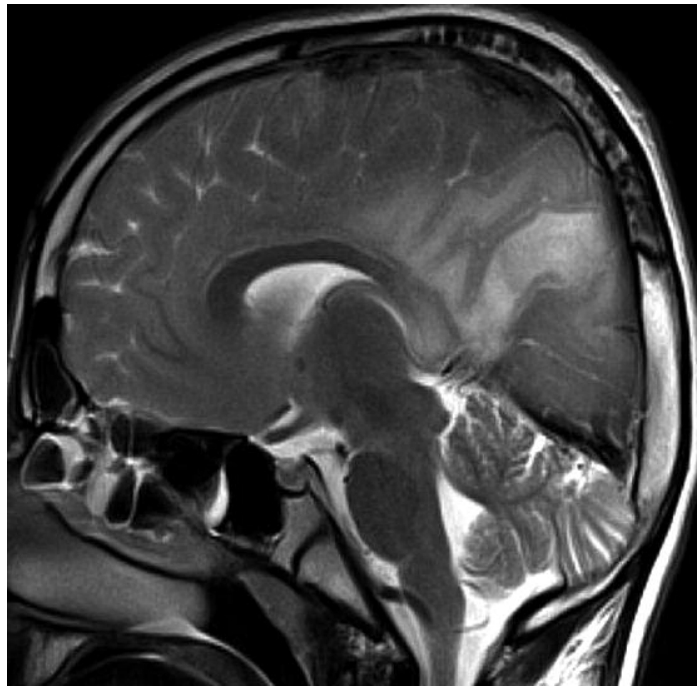

Figure 1. Sagittal T2-weighted images show widespread hyperintensities in cortical-subcortical and deep periventricular white matter of right parietooccipital region and in splenium of corpus callosum

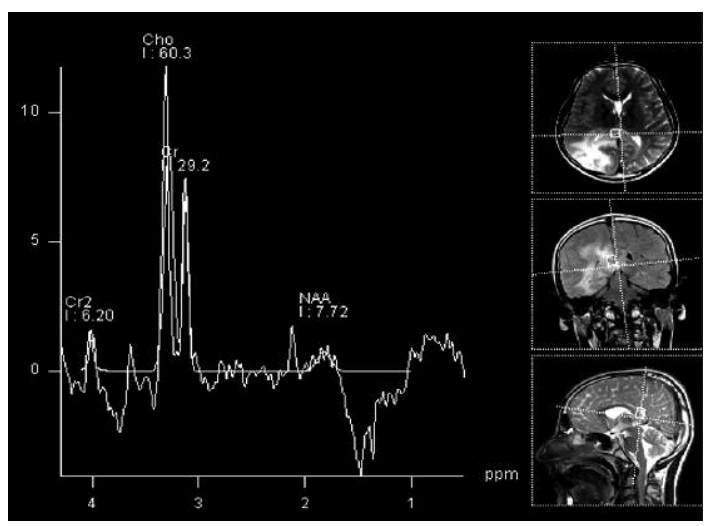

Figure 2. Multivoxel MRS (TE: $135 \mathrm{~ms}$ ) obtained from splenium of corpus callosum reveal prominent decreased NAA/Cr, increased $\mathrm{Cho} / \mathrm{Cr}$ and presence of lactate at $1.33 \mathrm{ppm}$ (inverted double)

not correlated. It is not possible to define these findings as improvement or progression based on the $M R I$ findings alone. Despite the progress of SSPE, improvement of MRI findings may be seen [2-5].

It has been reported that histopathological examinations show evidence of neuronal loss, astrogliosis, demyelination, and infiltration of inflammatory cells [5]. Magnetic resonance spectroscopy can provide information on neuronal and axonal viability, cellular energetics, and membrane status. In our case, MRS showed significantly decreased NAA/Cr ratios in the right parietooccipital subcortical white matter and splenium of the corpus callosum. The MR spectrum of the corpus callosal splenial lesion revealed increased $\mathrm{Cho} / \mathrm{Cr}$ and presence of lactate. These findings might indicate neuronal loss, demyelination, active inflammation and/or anaerobic metabolism. Magnetic res- onance spectroscopy findings were consistent with stage III SSPE [5]. Although to date SSPE is not completely curable, with early diagnosis it is possible to slow the progression of the disease.

Although MRS is not specific or diagnostic, it can show metabolic changes in the early-late stage [7]. For this reason, MRS may be useful to reveal the extent and progression of SSPE.

\section{References}

1. Eroglu E, Gokcil Z, Bek S, Ulas UH, Ozdag MF, Odabasi Z. Long-term follow-up of patients with adult-onset subacute sclerosing panencephalitis. J Neurol Sci 2008; 275: 113-6.

2. Alkan A, Kutlu R, Sigirci A, Aslan M, Sarac K, Yakinci C. Subacute sclerosing panencephalitis with fulminating course: follow-up magnetic resonance spectroscopy (MRS) findings. J Child Neurol 2004; 19: 233-8.

3. Oguz KK, Celebi A, Anlar B. MR imaging, diffusionweighted imaging and MR spectroscopy findings in acute rapidly progressive subacute sclerosing panencephalitis. Brain Dev 2007; 29: 306-11.

4. Garg RK, Anuradha HK, Varma R, Singh MK, Sharma PK. Initial clinical and radiological findings in patients with SSPE: are they predictive of neurological outcome after 6 months of follow-up? J Clin Neurosci 2011; 18: 1458-62.

5. Alkan A, Sarac K, Kutlu R, et al. Early- and late-state subacute sclerosing panencephalitis: chemical shift imaging and single-voxel MR spectroscopy. AJNR Am J Neuroradiol 2003; 24: 501-6.

6. Cakmakci H, Kurul S, Iscan A, Dirik E. Proton magnetic resonance spectroscopy in three subacute sclerosing panencephalitis patients: correlation with clinical status. Childs Nerv Syst 2004; 20: 216-20.

7. Teksam A, Cakır B, Ağildere AM. Proton MR spectroscopy in the diagnosis of early-stage subacute sclerosing panencephalitis. Diagn Interv Radiol 2006; 12: 61-3.

8. Cece H, Tokay L, Yildiz S, Karakas O, Karakas E, Iscan A. Epidemiological findings and clinical and magnetic resonance presentations in subacute sclerosing panencephalitis. J Int Med Res 2011; 39: 594-602. 